\title{
PECULIARITIES OF SPACE-CHARGE-LIMITED PHOTOCURRENT
}

\author{
M. Viliūnas, G. Juška, K. Arlauskas and V. Baltušis \\ Department of Solid State Electronics, Vilnius University \\ Sauletekio 9, III build., 2054 Vilnius, Lithuania
}

\begin{abstract}
We demonstrate new advantages of the space-charge-limited photocurrent technique for the investigations of charge carrier recombination. Bimolecular recombination coefficient in a-Si:H estimated according to suggested method for both electrons and holes is presented.
\end{abstract}

PACS numbers: 73.50.Fq, 73.50.Gr, 73.61.Jc

In a time-of-flight (TOF) experiment, nonequilibrium carriers are generated by short electrical or optical pulse in thin semi-insulating sample, sandwiched between two electrodes with applied external dc electric field. As a result, transient current occurs, and from its dynamics peculiarities of carrier transport can be studied.

The TOF technique can be used in the so-called "small-signal" regime, for which the charge of nonequilibrium carriers is smaller than the charge on the external electrodes $\left(Q \ll C U_{0}\right.$, where $C$ is the sample capacity and $U_{0}$ is the applied voltage). If $Q>C U_{0}$, space-charge-limited current (SCLC) results. Standard SCLC theory, describing injection current peculiarities is presented in [1]. Also, the SCLC regime is achievable for optical carrier generation by powerful laser pulses (photo-SCLC regime), and can be used, with some limitations [2], to study the density-of-states [3] and bimolecular recombination [4] in disordered materials. However, it must be mentioned that comprehensive interpretation of the photo-SCLC regime has not been worked out so far. In this contribution we continue our previous studies $[4,5]$ and present detailed theoretical analysis of the photo-SCLC regime. The results of our analysis are compared with data, obtained from photo-SCLC measurements in amorphous hydrogenized silicon a-Si:H.

In analyzing the photo-SCLC regime, we use the following assumptions of the standard SCLC theory: the one-dimensional description of the sandwich-like sample geometry, uniform initial distribution of the electric field, and negligible influence of thermal carrier generation. Carrier trapping, monomolecular recombination and diffusion are neglected. The carriers are generated by instantaneous light pulse. The bimolecular recombination as well as different resistivities of the detection resistor are taken into account. We solve the total current equation

$$
j(t)=\left[n(x, t)+\frac{\mu_{p}}{\mu_{n}} p(x, t)\right] E(x, t)+\frac{\partial E(x, t)}{\partial t}=\frac{1-U(t)}{R},
$$


Poisson's equation

$$
\frac{\partial E(x, t)}{\partial x}=p(x, t)-n(x, t),
$$

and kinetic equations for bimolecular recombination

$$
\frac{\partial p(x, t)}{\partial t}=\frac{\partial n(x, t)}{\partial t}=-B p(x, t) n(x, t)
$$

with initial conditions

$$
E(x, 0)=1, \quad n(x, 0)=p(x, 0)=L \alpha \exp (-\alpha x),
$$

and boundary condition

$$
\int_{0}^{1} E(x, t) \mathrm{d} x=1 \text {. }
$$

All equations use dimensionless units (coordinate $x$, time $t$ and so on), normalized to their characteristic parameters as is described below. Thus, the coordinate $x^{\prime}$ is normalized to the sample thickness $x=x^{\prime} / d$, the time $t^{\prime}$ is normalized to the small signal transit time $t=t^{\prime} / t_{\mathrm{tr}}=t^{\prime} \mu_{n} U_{0} / d^{2}$, the surface density of photogenerated $\mathrm{e}-\mathrm{h}$ pairs $L^{\prime}$, and the carrier concentrations $n^{\prime}$ and $p^{\prime}$ are normalized to the value of the initial charge on the electrodes: $L=L^{\prime} e d / \varepsilon \varepsilon_{0} U_{0}, n=n^{\prime} e d^{2} / \varepsilon \varepsilon_{0} U_{0}, p=$ $p^{\prime} e d^{2} / \varepsilon \varepsilon_{0} U_{0}$. Accordingly, in the small signal regime $L \ll 1$, while in the SCLC regime $L>1$. The electric field $E^{\prime}$ is normalized to its initial value $E=E^{\prime} d / U$, the current $j^{\prime}$ is normalized to the standard SCLC current $j=j^{\prime} d^{3} / \varepsilon \varepsilon_{0} \mu_{n} U_{0}^{2}$, and the bimolecular recombination coefficient $B^{\prime}$ is normalized to the diffusion-controlled recombination (Langevin) value $B=B^{\prime} / B_{\mathrm{L}}=B^{\prime} \varepsilon \varepsilon_{0} / e \mu_{n}$. The absorption coefficient $\alpha=\alpha^{\prime} d$ is normalized to the inverse sample thickness $d$. The resistivity of the registration circuit is $R=R^{\prime} \mu_{n} \varepsilon \varepsilon_{0} U_{0} / d^{3}$. Hence, for $R<1$ we have differential SCLC regime, which is governed by the sample resistivity. For $R>1$ we have integral SCLC regime, where the current is determined by the load resistor.

Figure 1 shows typical features of differential mode photo-SCLC transients calculated for electrons (Fig. 1, top) and holes (Fig. 1, bottom) with the same bimolecular recombination coefficient, drifting from the illuminated electrode for various e-h pair densities $L$. Standard SCLC transient [1] as well as transient for $L=1000, B=0$ are presented for comparison. Carrier mobility ratio $\mu_{n} / \mu_{p}=100$ is assumed. In the following we must distinguish between the two cases where either the electrons or the holes drift across the sample. Due to different carrier mobility, the bimolecular recombination has different influence on the electron and hole transients. In both cases the bimolecular recombination goes on until the electron and hole reservoirs stay together. As a result, the recombination lasts approximately 100 times longer for the hole drift than for the electron drift. Hence, for the hole drift the bimolecular recombination will be more effective. However, repeating our calculations for the holes, and using dimensionless units normalized to the hole mobility, yields $B_{\mathrm{h}}=1$ (Langevin case). As a consequence, for the hole drift there is no possibility to extract charge larger than $C U_{0}$ regardless of the generation intensity. Since the half magnitude time $t_{1 / 2}$ (see definition below) for the holes does not chanje (Fig. 1, bottom), determination of the bimolecular recombination coefficient for $B \geq 1$ becomes impossible. For electrons, in contrast, 


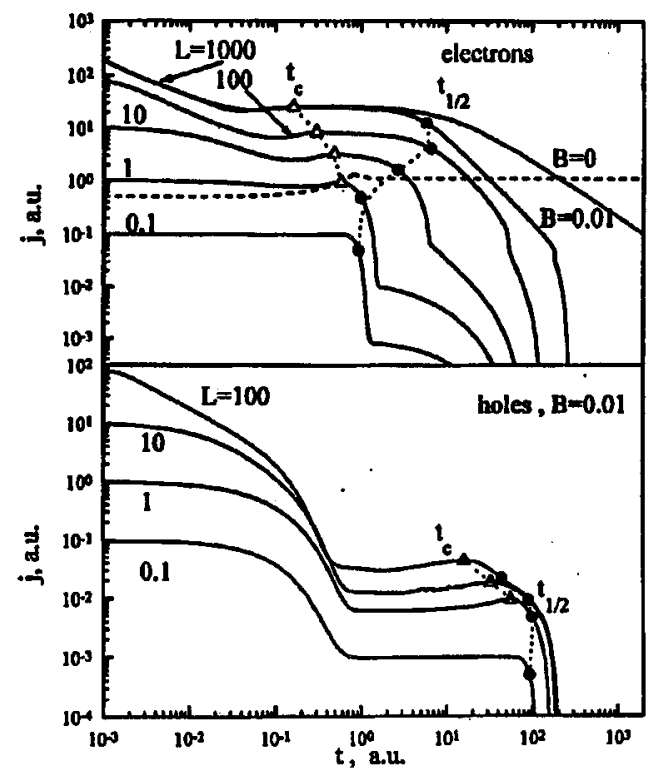

Fig. 1. The calculated electron (top) and hole (bottom) photo-SCLC transients for different light intensities $L(\alpha=10, R=0, B=0.01)$ in comparison with standard SCLC and $L=1000, B=0$ case.

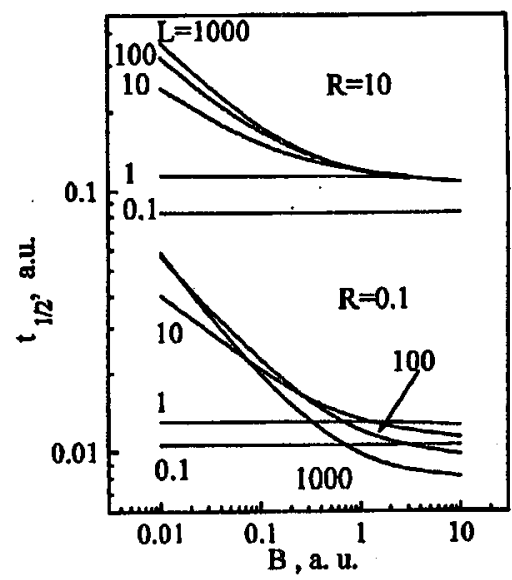

Fig. 2. The hole drift half magnitude time $t_{1 / 2}$ dependencies on bimolecular recombination coefficient $B$ for different light intensities $L(\alpha=20)$.

$t_{1 / 2}$ increases with excitation intensity, and then saturates according to the value of $B$.

Figure 2 shows calculated hole's TOF half-magnitude time $t_{1 / 2}$, defined as a time when $j \geq j\left(t_{\mathrm{c}}\right) / 2$ for differential mode (Fig. 1$)$, or $j \geq j_{\max } / 2\left(j_{\max }\right.$ is maximum current) for integral mode [5], as a function of bimolecular recombination 
coefficient (normalized to the electron mobility) for different light intensity in both integral and differential operating modes. For differential mode $(R=0.1)$ we obtained no saturation of $t_{1 / 2}$ with increasing generation intensity in contrast to the integral mode $(R=10)$ where the saturation is clear. This is because the effective sample thickness becomes reduced by the length of the photoinduced carrier reservoir $d_{\mathrm{L}}=\ln (L) / \alpha$, and the differential operating mode extraction current increases, leading to the decrease in $t_{1 / 2}$. In experiments, especially for the case of high carrier mobility, the integral mode of TOF is usually used. In this case the $j(t)$ transients are not as expressive as in differential mode [5], however, the above mentioned peculiarities occur as well. Since the dependence $j(L)$ is weak, the integral mode offers some advantages for the recombination studies.

By fitting the experimentally obtained $t_{1 / 2}(L)$ dependence with theoretical results calculated for different $B$, it is possible to evaluate the bimolecular recombination coefficient. Figure 3 presents the temperature dependence of the bimolec-

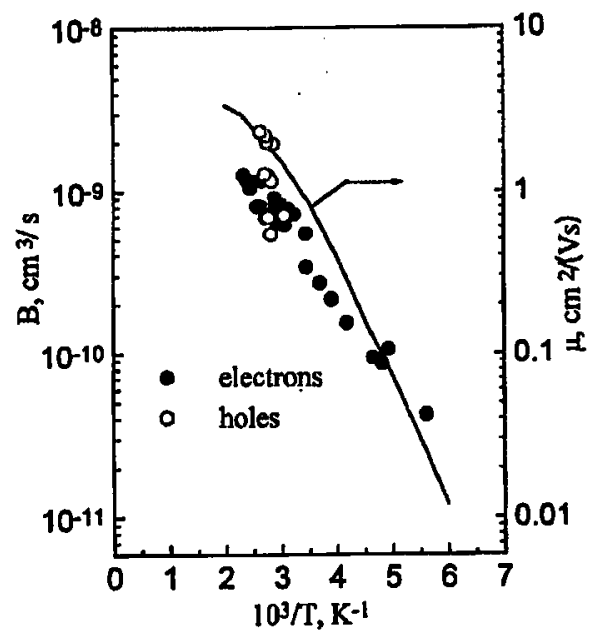

Fig. 3. Temperature dependencies of the electron drift mobility (solid line) and bimolecular recombination coefficient (points).

ular recombination coefficient estimated from experimentally measured saturation values of $t_{1 / 2}$ for electrons [6] and holes in a-SiH. For comparison the electron drift mobility activation curve is given in the same plot. The bimolecular recombination coefficient, obtained from hole TOF measurements demonstrates good agreement with the same data for the electrons, although their values of $B$, normalized to the carrier mobility, are different. This causes bimolecular recombination coefficient (normalized to the hole mobility) $B_{\mathrm{h}}>1$ at room temperatures. Similarity between the temperature dependencies of the bimolecular recombination coeffcient and the electron mobility demonstrates that the bimolecular recombination is governed by carrier diffusion, as one would expect in the material with short mean free path. 
The values of bimolecular recombination coefficient, evaluated from the photo-SCLC measurements are in good agreement with the same values obtained by optical pump-and-probe experiments $[6,7]$. In comparison with the latter technique, photo-SCLC method provides some advantages: the measurements are carried out in the nanosecond time scale although the recombination occurs in the picosecond time scale; also there is a possibility to measure $B$ separately for the electrons and holes, and to distinguish between carrier trapping and recombination processes.

In conclusion, we performed theoretical analysis of the photo-SCLC regime in TOF experiments. We demonstrated how this technique can be used for the ultrafast recombination investigations in semi-insulating semiconductors, and pointed out some advantages of this technique over the optical measurements. We compared for the first time the bimolecular recombination coefficients, obtained from the electron and hole transients according to the results of our analysis. As expected, the same results were obtained in both cases.

\section{References}

[1] A. Many, G. Rakavy, Phys. Rev. 126, 1980 (1962).

[2] G. Juška, M. Viliūnas, K. Arlauskas, A. Fejfar, J. Kočka, in: 23rd Int. Conf. on the Physics of Semiconductors, Eds. M. Scheffler, R. Zimmermann, Vol. 1, World Scientific, Singapore 1996, p. 125.

[3] F. Schauer, A. Eliat, M. Nesladek, G.J. Andriaenssens, Appl. Phys. Lett. 64, 3009 (1994).

[4] G. Juška, M. Viliūnas, O. Klima, E. Šipek, J. Kočka, Philos. Mag. B 69, 277 (1994).

[5] G. Juška, M. Viliūnas, K. Arlauskas, J. Kočka, Phys. Rev. B 51, 16668 (1995).

[6] G. Juška, K. Arlauskas, J. Kočka, Lith. Phys. J. 35, 611 (1995).

[7] P.M. Fauchet, D. Hulin, R. Vanderhaghen, A. Mourchid, W.L. Nighan Jr., J. Non-Cryst. Solids 141, 76 (1992). 